\title{
Performance Analysis of a BiNMOS Device
}

\author{
J. B. Kuo*, G. P. Rosseel, R. W. Dutton \\ Staniord University \\ *Dept. of Electrical Eng. \\ National Taiwan University, Taipei, Taiwan
}

Abstract

The BiNMOS device associated with $2 \mu \mathrm{m}$ n-well BiCMOS technologies consumes substantial area for isolation. A merged BiNMOS device structure is introduced to reduce the device size. The periormance of the merged BiNMOS device has been analyzed by PISCES-2B(1). DC characteristics show the merged BiNMOS device has a higher driving capability. Turn-on transient analysis shows substantial substrate currents during transient.

\section{Summary}

Figure la shows a BiCMOS inverter. The NMOS and bipolar devices are the most important part during the pull-down transient $(2,3,4)$. The BiNMOS device in the $2 \mu \mathrm{m}$ BiCMOS structure without a buried layer as shown in Figure $1 b$ is composed of bipolar and NMOS devices with substantial space for isolation between the two devices. Between the source and the n-well regions, there is $\mathrm{P}+$ channel stop area. Source contact of the NMOS device and base contact of

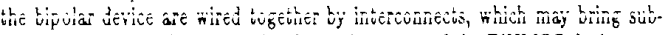
stantial parasitics. Corsequently, the performance of the BiNMOS device may be degraded. In this paper, a BiNMOS device structure with a butting source and collector area is introduced to reduce the device size. The merged BiNMOS device as shown in Figure $1 \mathrm{c}$ is based on a $2 \mu \mathrm{m}$ BiCMOS technology without a buried layer. The drain nf the NMOS device and the collector contact area of the bipolar device are placed against each other with a shared electrode. This arrangement shrinks the length of the BiNMOS device owing to the elimination of the $\mathrm{P}+$ channel stop area. The distance between the $\mathrm{N}+$ collector area and the base/collector junction at surface is about $0.8 \mu \mathrm{m}$. The NMOS device has an effective channel length of $1.4 \mu \mathrm{m}$ and the bipolar one has a base width of $0.26 \mu \mathrm{m}$ and a peak concentration of $2 \times 10^{17} \mathrm{~cm}^{-9}$. The doping concentrations of the $n$-well and the p-substrate are $1 \times 10^{16} \mathrm{~cm}^{-9}$ and $5 \times 10^{15} \mathrm{~cm}^{-3}$ respectively.

DC analysis has been done for the merged BiNMOS and the standard one. The drain contact of the NMOS device and the collector contact of the bipolar device are connected together as the collector electrode. The source contact of the NMOS device and the base contact of the bipolar device are connected by a common electrode. The substrate of the NMOS device is connected to the back gate via a $100 \mathrm{~K} \Omega$ resistance accounting for the effective resistance in the neutral substrate region. A $1 \times 10^{12} \Omega$ registor is placed between ground and the base/source node to avoid the convergence problems for $\mathrm{DC}$ analysis. Both Jevices have an identical channel length. Due to the merged drain and collector contact structure, the merged device has a larger emitter width of $0.7 \mu \mathrm{m}$. Applying a bias between the gate and the emitter electrode $\left(V_{G}\right)$ from $0 \mathrm{~V}$ to $5 \mathrm{~V}$ and a collector/drain-to-emitter bias $\left(V_{C E}\right)$ from $5 \mathrm{~V}$ to $1 \mathrm{~V}$, the absolute values of the emitter currents in amp/ $\mu \mathrm{m}$ have been obtained as shown in Figure $2 \mathrm{a}$. Solid lines show the case with the merred BiNMOS device and the dashed ones with the standard one. The merged BiNMOS device has a threshold voltage of $1.6 \mathrm{~V}$, which is defined as the $\mathrm{V}_{\mathrm{G}}$ as the magnitude of the emitter current reaches $0.1 \mathrm{~mA}$, at a $V_{C E}$ of $5 \mathrm{~V}$. A smaller $V_{C E}$ increases the threshold voltage due to the smaller drain-to-source voltage and the corresponding smaller emitter current. The standard BiNMOS one shows a larger threshold voltage(1.7V) because of the smalier emitter width of $0.5 \mu \mathrm{m}$. After the devices turn on, the merged BiNMOS device does have higher currents due to more area has been ailocated for intrinsic bipolar area. In fact, the standard device may have a worse performance since the cross section used in the analysis does not include the structure for collector contact. For $V_{\mathrm{CE}}$ from $5 \mathrm{~V}$ to $2 \mathrm{~V}$, the magnitude of the enitter current changes accordingly with $V_{G}$ and $V_{C E}$. For the $V_{C E}$ of IV, the bipolar device saturates. Consequently, an increase in $V_{G}$ changes the emitter current sightly. Figure $2 b$ shows the corresponding base-to-emitter vollafe $\left(V_{B E}\right)$ as a function of $\left(V_{G}\right)$ for the two devices with $V_{C E}$ changing from
$1 V$ to $5 \mathrm{~V}$ at a step of $1 \mathrm{~V}$. Solid lines show the case for the merged device and the dashed ones for the standard one. At the threshold voltage(1.6V) of the mierged derice, $V_{B E}$ is about $0.75 \mathrm{~V}$, which indicates the bipolar device is also turned on. For the standard device, the $V_{B E}$ vs. $V_{G}$ curves match well with the merged one's before the turn-on of the device. After turn-on, the larger $V_{B E s}$ of the merged one at the same $V_{G} s$ are due to surface contact structure for the collector.

Pull-down transient has been obtained for the merged devices with appropriate boundary conditions. An output load capacitance of $0.2 \mathrm{pf}$ is connected to the collector and a capacitance of $0.01 \mathrm{fF}$ accounting for parasitics is at the base/source node. By imposing a voltage step from $0 \mathrm{~V}$ to $5 \mathrm{~V}$ in 0. lns at the input, the pull-down transient behavior of the merged BiNMOS device has been obtained. This required about 28 hours on a Convex C-1 computer using vectorized enhancements. In order to show the role of the neutral substrate, the merged device with a $100 \mathrm{~K} \Omega$ contact resistance at the substrate electrode has also been studied. The terminal currents and voltages for the device with and without the $100 \mathrm{~K} \Omega$ substrate contact resistance during the pull-down transient are shown in Figure 3 . Solid lines are for the device without the $100 \mathrm{~K} \Omega$ contact resistance at the substrate electrode and the dashed lines are with it. Both devices show similar transient behavior for the terminal voltages and currents except for the substrate current. Initially, the input voltage is at $\mathrm{OV}$ and the output yoltage is at $5 \mathrm{~V}$. During the ramp up period, after the NMOS device turns on, both base/drain voltages go up quickly, the slopes being determined by the parasitic capacitance at the base/source node and the NMOS charging currents, until the bipolar devices turn on. During this period, the magnitude of the coilector currents increases. After $0.15 n$, the bipolar transistors become charged. Consequently, collector currents are increasing more slowly and the output voltages fall. After $2.1 \mathrm{~ns}$, the bipolar devices are saturated. Therefore, the collector currents are decreasing and the output voltages are decreasing more slowly. At the same time, the base/source voltages begin to fall due to the removal of the mobile carriers in the virtual base area. Finally at $6 \mathrm{~ns}$, the base/source voltages decrease to about $0.12 \mathrm{~V}$ and the corresponding $\mathrm{V}_{C E}$ s are less than $0.02 \mathrm{~V}$. The terminal currents at the NMOS substrate electrode act quite differently for the devices with and without substrate contact resistance. During the input ramp up period, substantial substrate currents owing to charge pumping exist for both cases. The device with the substrate contact resistance has a higher charge pumping currents. As the bipolar device becomes saturated, the device with the substrate contact resistance has a lower current at the substrate electrode.

More insight into device operation can be obtained by examining the change in total electrons during transient. Figure 4 showg the absolute change of the total electron concentration and the total recombination rates outside the $\mathrm{n}$ - $\mathrm{well}$ region. The initial rate of change in the total electrons in the NMOS region is large due to the build-up of the NMOS channel during the input ramp-up period. After that, the rate of change remains constant until the bipolar device becomes saturated. Finally, the rates of change in total electrons become negative. Before saturation of the bipolar device, the recombination in the p-substrate area is negligible. After that, substantial recombination can be observed due to the injection of the electrons into the p-substrate as shown in Figure 5. The recombinations reach peak as the substrate currents are at the maximum. The device with the substrate contact resistance does have a higher injection of electrons in the p-substrate region as shown in Figure 5. Consequently, the recombination rate is also higher.

CH1234-5/89/0000-P1-4.1\$01.00@1989 IEEE P1-4.1 
Conclusion

A merged BiNMOS device structure is introduced to reduce the device size. The DC characteristics show the merged BiNMOS device has a higher driving capability. Turn-on transient analysis shows substantial substrate currents during transient.

\section{References}

1. M. R. Pinto, C. S. Rafiety, and R. W. Dutton, "PISCES 2: Poisson and Con. tinuity Equation Solver," Technical Report, Stanford University, Sept. 1984 2.H. D. L. Santos and B. Hoefflinger, "Optimization and Scaling of CMOSBipolar Drivers for VLSI Interconnects," IEEE Transaction on Electron Devices, pp.1722-1730, Vol. 33, Nov. 1986

3.E. W. Greeneich and K. L. McLaughin, "Analysis and Characterization of BiCMOS for High-Speed Digital Logic," IEEE Journal of Solid-State Circuits, pp.558-565, Vol. 23, April 1988

4. G. P. Rosseel et. al., "Deiay Analysis for BiCMOS Drivers," IEEE Proceedings of VLSI Circuit and Technology Symposium 1988

\section{Acknowledgments}

This work is supported under U.S. Army Research Office Contract \#DAAL0387-G.0076 and R.O.C. National Science Council Contract \#NSC77-0404-E002-

44.

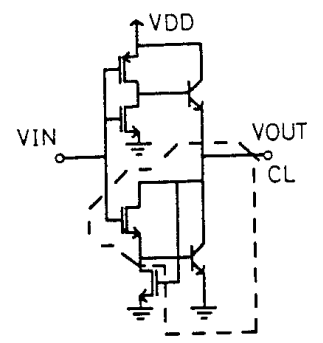

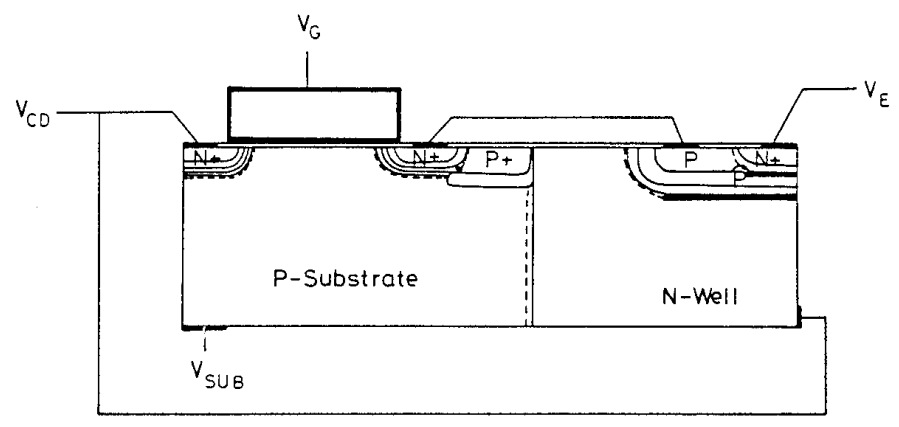

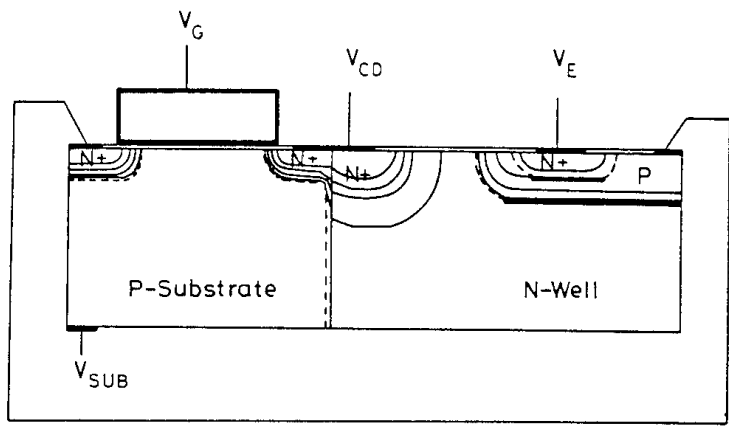

Figure 1. a. The BiCMOS inverter. b. Cross section of the conventional BirMOS device $c$. Cross section of the merged BiNMOS device 

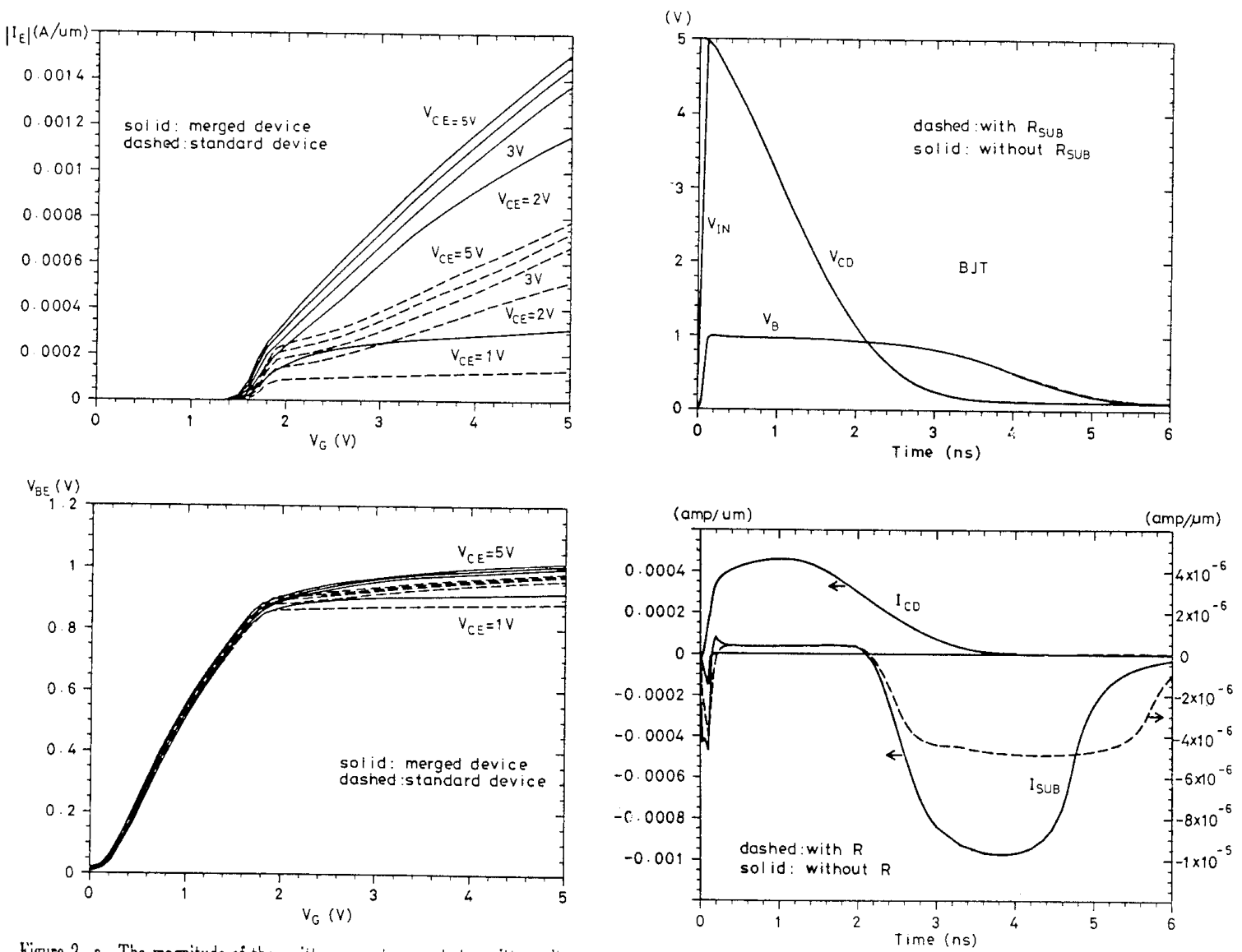

Figure 2. 2. The magnitude of the emitter current vs. gate-to-emitter voltage curves with $V_{C E}=1 \mathrm{~V}-5 \mathrm{~V}$ for the merged and standard BiNMOS devices $b$. The base-to-emitter voitage vs. gate-to-emilter voitage curves for

Figure 3. Terminal currents and voltages during transient $V_{C E}=1 V-5 V$ for the merged and standard Bikivos uevices

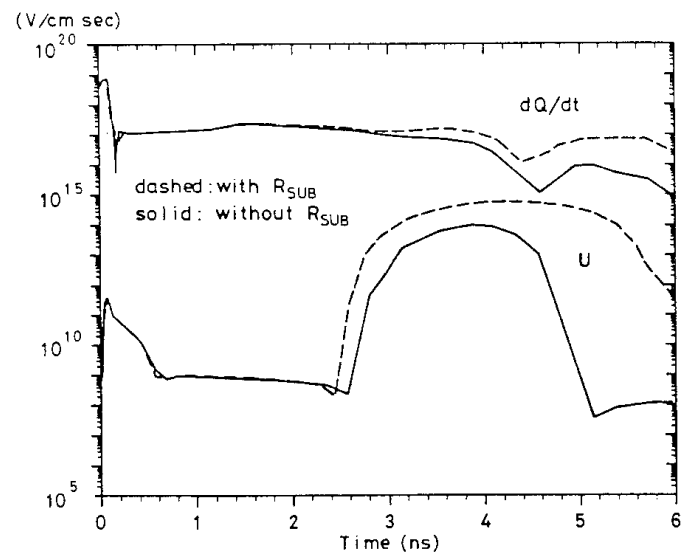

Figure 4 . The absolute rate of change in the total electrons $\left.\frac{d Q_{n}}{d t}\right)$ and the absolute total recombination rate, $U\left(\mathrm{~cm}^{-1} \mathrm{sec}^{-1}\right)$ in the p-substrate region

$$
\mathrm{P} 1-4.3
$$



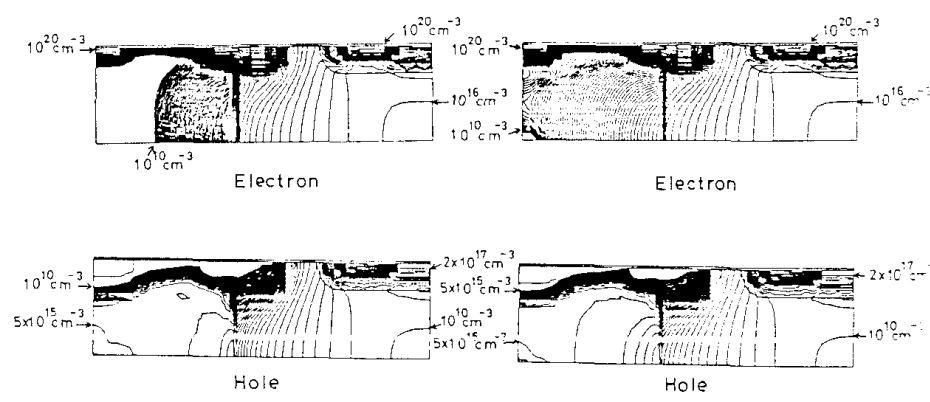

(a)

(b)

Figure 5, an Electron and hole concentration contours for the device without

the lookn substrate contact resistance at 3ns b.Etectron and hole concentra-

tion contours for the device with the $100 \mathrm{~K} \Omega$ substrate contact resistance at $3 \mathrm{~ns}$

P1-4.4 